\title{
Apports Du Modele Numerique De Terrain Mnt A La Modelisation Du Relief Et Des Caracteristiques Physiques Du Bassin Versant Du Moyen Beht En Amont Du Barrage El Kansera (Sillon Sud Rifain, Maroc)
}

\author{
Ahmed Laabidi, Docteur \\ Université Sidi Mohamed Ben Abdellah, Faculté des Sciences, Dhar El \\ Mehraz, Département de Géologie, Fès, Maroc.
}

Abdellah El Hmaidi, PES

Université Moulay Ismail, Faculté des Sciences de Meknès, Département de Géologie, B.P. 11201, Zitoune, Meknès, Maroc.

\section{Lahcen Gourari, PES}

Université Sidi Mohamed Ben Abdellah, Faculté des Sciences, Dhar El Mehraz, Département de Géologie, Fès, Maroc.

\section{Mourad El ABASSI, Master}

Université Moulay Ismail, Faculté des Sciences de Meknès, Département de Géologie, B.P. 11201, Zitoune, Meknès, Maroc.

doi: 10.19044/esj.2016.v12n29p258 URL:http://dx.doi.org/10.19044/esj.2016.v12n29p258

\begin{abstract}
The watershed of the Beht River occupies the southwest part of the Sebou watershed with an area of 4,603.77 km2. It starts in the Causse of Middle Atlas, drains the northern edge of central Morocco and ends in the rifan south wrinkles at the level of dam El Kansera. The geographical information system (GIS) and ASTER images with a resolution of 30m have been of great use for creating thematic maps and automatic extraction of a multitude of parameters and physiographic indices of this watershed. These parameters, very characteristic, allow to assess the weight of the geomorphology on surface water flow and the influence of the latter by the risk of erosion and of flooding which have a very negative consequences on human and material plan, at the level of the studied watershed.
\end{abstract}

Keywords: Watershed, Beht river, El Kansera dam, Physical characteristics, DEM 


\section{Résumé}

Le bassin versant de l'Oued Beht, d'une superficie de 4603,77 Km², occupe la partie sud-ouest du bassin versant du Sebou. L'Oued Beht prend naissance dans le Causse moyen atlasique, draine la bordure septentrionale du Maroc central et se jette dans le barrage El Kansera au niveau des rides sud-rifaines. Les SIG et les images ASTER, d'une résolution de $30 \mathrm{~m}$, ont été d'une grande utilité pour la création de cartes thématiques et l'extraction automatique d'une multitude de paramètres et d'indices physiographiques de ce bassin versant. Ces paramètres, très caractéristiques, permettent d'évaluer le poids de la géomorphologie sur l'écoulement des eaux superficielles et l'influence de ce dernier par le risque d'érosion et d'inondations à conséquences très négatives sur le plan humain et matériel au niveau du bassin versant étudié.

Mots clés: Bassin versant, Oued Beht, Barrage El Kansera, caractéristiques physiques, MNT

\section{Introduction}

Le bassin versant (BV) ou bassin hydrologique est le territoire qui recueille les eaux de ruissellement et d'infiltration alimentant soit un fleuve, une rivière ou une ravine. Il les concentre vers le point de sortie appelé exutoire. Le bassin versant est défini par le relief et délimité par les lignes de partage des eaux (lignes de plus hautes altitudes qui déterminent la direction d'écoulement des eaux de pluie). Le sous-bassin (SB) représente le bassin versant d'un affluent. L'exutoire d'un bassin est le point le plus en aval du réseau hydrographique par lequel passent toutes les eaux de ruissellement drainées par le bassin. La ligne de crête d'un bassin versant est la ligne de partage des eaux. Cette ligne ainsi définie, limite les bassins versants topographiques adjacents (Morell et al., 1999).

Les techniques classiques utilisées dans l'étude du complexe physique d'un bassin versant sont basées essentiellement sur des méthodes manuelles, dont les résultats obtenus généralement sont incorrects. Avec l'apparition des nouveaux outils tels que les SIG et la télédétection, il est devenu facile de déterminer les paramètres de forme et de relief d'un bassin versant.

L'utilisation de ces nouvelles techniques permettra ainsi de répondre aux exigences d'exploitation des eaux superficielles et de la prévention contre les risques d'inondation. L'objectif de ce travail se limite à la caractérisation des aspects morphométriques et du relief du bassin versant de l'Oued Beht moyen situé en amont du barrage El Kansera, du fait que ces 
paramètres jouent un rôle déterminant dans l'écoulement des bassins versants et dans les risques d'érosion.

L'analyse morphométrique est une démarche importante pour la hiérarchisation des bassins versants même sans tenir compte de la carte des sols (Biswas et al., 1999). Pour ce faire, un ensemble de paramètres quantitatifs importants sont utilisés dans la compréhension du dynamisme d'une rivière et de son bassin versant comme la superficie, le périmètre, la longueur, la largeur, l'indice de compacité de Gravelius, l'indice de pente, la pente moyenne, l'altitude maximale et l'altitude minimale, etc. (Veltri et al., 1996). Ceux-ci ont été obtenus à partir du MNT de la zone d'étude.

\section{Présentation du bassin versant de l'Oued Beht en amont du barrage El Kansera}

L'Oued Beht est l'un des principaux oueds du domaine atlantique marocain. Il prend ses sources dans la bordure occidentale du Causse moyen atlasique et draine la bordure septentrionale du Maroc Central. A sa sortie de ce domaine, il traverse le Couloir Sud Rifain. Dans la partie Sud de la plaine du Gharb, à l'aval du barrage EL Kansera, l'Oued Beht reçoit les eaux de l'Oued Rdem et Oued Tifelt avant de rejoindre l'Oued Sebou au niveau de la confluence de Morgane (Fig.1, ABH, 2011).

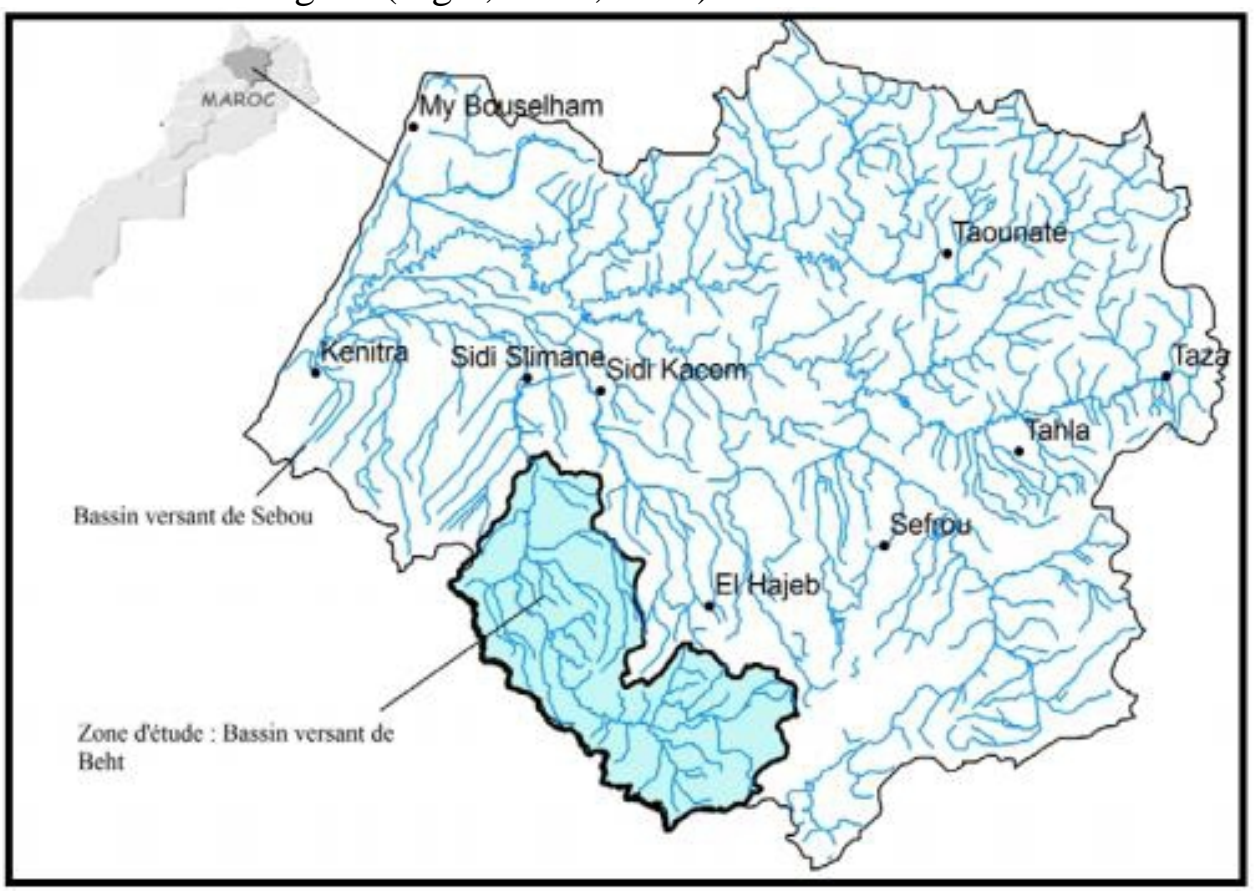

Figure 1. Situation géographique du Bassin versant de l'Oued Beht dans le contexte du bassin versant de l'Oued Sebou (ABH, 2011). 
Le bassin versant de l'Oued Beht occupe la partie Sud-Ouest du bassin de Sebou. Il est limité au Nord par la plaine du Gharb et le plateau de Meknès, au Sud par le bassin de l'Oum Er-Rbia, à l'Ouest par le bassin de Bouregrag et à l'Est par le Moyen Atlas (Fig.1).

\section{Méthodologie et données utilisées}

Dans le cadre de cette étude, le Modèle Numérique de Terrain (MNT) utilisé provient des images SRTM (Shuttle Radar Topography Mission), prises le 15 Mars 2011. Ce MNT, d'une résolution de 30*30m, a été utilisé pour l'extraction des différents paramètres physiographiques du bassin versant de l'Oued Beht en amont du barrage El Kansera.

Les études morphologique et physiographique nécessitent, entre autres, l'analyse des paramètres tels que la structure et la forme du bassin versant. A partir du MNT, le bassin versant étudié peut être délimité avec précision avec extraction automatique des différents paramètres caractéristiques de son relief. Pour développer cette démarche, les logiciels dédiés au SIG (Système d'Information Géographique) sont très nécessaires.

Les caractéristiques morphologiques (surface, longueur, largeur, périmètre, forme, relief, pente) et les indices morphométriques du bassin versant sont indispensables pour caractériser son environnement physique et leurs influences sur l'écoulement superficiel. Ces facteurs, de nature purement géométrique ou physique, ont été extraits facilement par des techniques automatiques en faisant appel aux techniques digitales des SIG et aux modèles numériques de terrain MNT.

\section{Résultats et discussion}

\section{Caractéristiques géométriques (morphologiques)}

Les principales caractéristiques physiques étudiées pour tout bassin hydrologique constituent des paramètres d'entrée des formules et des modèles de calcul pour la caractérisation des bassins versants.

Les caractéristiques physiographiques d'un bassin versant influencent fortement sa réponse hydrologique, et notamment le régime des écoulements en période de crue ou d'étiage (Biswas, 1999, Michel, 1973). Le temps de concentration Tc, qui caractérise en partie la vitesse et l'intensité de la réaction du bassin versant à une sollicitation des précipitations, est influencé par diverses caractéristiques morphologiques : en premier lieu, la taille du bassin (sa surface), sa forme, son relief, sa pente longitudinale et son orientation. A ces facteurs s'ajoutent encore la lithologie des terrains et la nature des sols, le couvert végétal et les caractéristiques du réseau hydrographique. Ces facteurs, d'ordre purement physique, s'estiment 
aisément à partir de cartes adéquates ou en recourant à des techniques digitales et aux modèles numériques.

Le bassin versant marque à la fois la notion topographique de zone limitée par une ligne de partage des eaux et celle de surface d'interception des précipitations susceptibles d'être drainées jusqu'à l'exutoire par le réseau hydrographique (FAO 1996).

\section{Surface et périmètre}

La superficie du bassin versant, ou surface drainante, est obtenue par planimétrie sur cartes topographiques où, comme dans ce travail, à l'aide des logiciels de cartographie par des techniques de digitalisation et de limitation. Le bassin versant étant l'aire de réception des précipitations et d'alimentation des cours d'eau, les débits vont être en partie reliés à sa surface.

Le périmètre du bassin versant est obtenu par curvimètre sur cartes topographiques ou à l'aide de logiciels informatiques comme dans le présent travail.

Ces deux paramètres, superficie et périmètre, apportent des informations précieuses dans l'étude des bassins versants. Leur influence se manifeste dans la nature de la relation entre le débit et le temps.

En ce qui concerne le bassin versant du Moyen Beht, il s'étale sur une superficie $A=\mathbf{4 6 0 3 , 7 7} \mathbf{k m}^{2}$ et présente un périmètre $P=\mathbf{4 2 1 , 4 3} \mathbf{~ k m}$.

\section{Indice de compacité de Gravelius, $K_{G}$}

L'indice de compacité de Gravelius (1914, In Roche 1963) $\mathrm{K}_{\mathrm{G}}$ est appelé également coefficient de forme (Strahler, 1964). Il est défini comme le rapport du périmètre du bassin versant $(\mathrm{P})$ au périmètre d'un cercle de même superficie. Il donne une idée sur la forme du bassin versant qui a une influence sur l'écoulement global du cours d'eau et surtout sur l'allure de l'hydrogramme (représentation graphique de l'évolution du débit $Q$ en fonction du temps t), résultant d'une pluie donnée, à l'exutoire du bassin. Par exemple, une forme allongée favorise, pour une même pluie, les faibles débits de pointes de crues, ceci en raison des plus importants temps d'acheminement de l'eau à l'exutoire. Ce phénomène est lié à la notion de temps de concentration. En revanche, les bassins en forme d'éventail, présentant un temps de concentration plus court, auront les plus forts débits de pointe.

L'indice $\mathrm{K}_{\mathrm{G}}$ ne peut être calculé, avec suffisamment de précision, que si le périmètre $\mathrm{P}$ est mesuré sur un contour de bassin très simplifié. Pour cela, il est nécessaire de supprimer toutes les sinuosités qui ne correspondent pas à la tête d'un thalweg actif. Pour vérifier que la stylisation du périmètre est suffisante, on s'assurera que la longueur du rectangle équivalent Leq n'est pas sensiblement supérieure à la longueur du plus long cours d'eau, depuis son 
origine jusqu'à l'exutoire (FAO, 1996). Si A est la surface du bassin en $\mathrm{km}^{2}$ et $\mathrm{P}$ son périmètre en $\mathrm{km}$, le coefficient $\mathbf{K}_{\mathbf{G}}$ est égal à :

$$
K \mathbf{G}=\frac{\mathrm{P}}{2 \cdot \sqrt{\pi \cdot \mathrm{A}}} \approx 0,28 \cdot \frac{\mathrm{P}}{\sqrt{\mathrm{A}}}
$$

Avec $\mathrm{A}=4603,77 \mathrm{~km}^{2}$ et $\mathrm{P}=421,43 \mathrm{~km}, \mathrm{~K}_{\mathrm{G}}=1,74$.

Cet indice est proche de 1 pour un bassin versant de forme quasiment circulaire (bien drainé) possédant un potentiel d'infiltration plus important que ceux de forme allongée. Il est égal à 1,12 pour un bassin versant de forme carrée et lorsqu'il devient supérieur à 1,12, le bassin est de forme allongée (Musy, 2005).

L'indice de compacité de l'Oued Beht est $\mathbf{K}_{\mathbf{G}}=\mathbf{1 , 7 4}$; ce bassin versant a donc une forme allongée. Sa direction principale d'allongement est Sud-Est-Nord-Ouest. Elle est parallèle au sens d'écoulement général des eaux. Cette forme favorise une érosion linéaire et régressive et les faibles débits de pointe de crue en raison du temps élevé de concentration ou d'acheminement de l'eau jusqu'à l'exutoire (Musy et Laglaine, 1992). Ce type de bassin versant devrait connaître très peu de cas d'inondation des localités riveraines même pendant les hautes eaux.

\section{Circularité du bassin}

La circularité d'un bassin versant Rc est le rapport entre sa surface et celle d'un cercle ayant le même périmètre que ce dernier (Miller, 1959). Elle s'exprime par :

$$
R c=\frac{4 \pi \cdot \mathrm{A}}{\mathrm{P}^{2}}
$$

Les valeurs de circularité du bassin s'approchant de 1 indiquent une forme quasi circulaire (Singh, 1992) et une pente relativement faible. Pour de tels bassins versants, l'infiltration est relativement uniforme et le temps que prend l'eau pour rejoindre l'exutoire est moins élevé que celui d'un bassin versant de forme allongée (Reddy et al., 2004). La circularité du bassin est davantage influencée par la longueur, la fréquence et la pente des affluents que par la pente du bassin versant et la forme du réseau de drainage. Un rapport significatif indique le stade dendritique du bassin versant. Les valeurs faibles, médianes et élevées sont des indices du stade (jeune, mature et vieux) du cycle de vie des bassins tributaires (Sreedevi et al., 2005).

Pour le bassin versant de l'Oued Beht, $\mathbf{R c}=\mathbf{0 , 3 3}$. Cette valeur faible témoigne d'une forme allongée du bassin. 


\section{Indice de forme de Horton, $\mathrm{K}_{\mathrm{H}}$}

L'indice de forme $\mathbf{K}_{\mathbf{H}}$ d'un bassin versant est la relation entre la surface du bassin $\left(\mathrm{km}^{2}\right)$ et la longueur du cours d'eau principal en $\mathrm{Km}$ (Horton, 1945). Il est inférieur à 1 pour un bassin versant de forme allongée et supérieur à 1 pour un bassin de forme ramassée.

Il est utilisé pour prédire l'intensité de l'écoulement à l'exutoire d'un bassin versant. Le facteur de forme montre une relation inverse avec la longueur du cours d'eau principal et possède une relation directe avec le débit de pointe à l'exutoire du bassin versant (Gregory et Walling, 1973). Un facteur de forme élevé $(>0,5)$ indique un BV de forme plutôt circulaire, et un débit de pointe élevé d'une durée relativement courte (Singh, 1992, Reddy et al., 2004).

$$
\mathbf{K}_{\mathbf{H}}=\mathbf{A} / \mathbf{L}^{2}
$$

Pour le bassin versant de l'Oued Beht, on a la surface $A=4603,77$ $\mathrm{km}^{2}$ et la longueur du cours d'eau principal $\mathrm{L}=167,56 \mathrm{~km}$. Donc l'indice de forme d'Horton, $\mathbf{K}_{\mathbf{H}}=\mathbf{0 , 1 6}$, est inferieur à 1 , et par conséquent, la forme du bassin versant est allongée et donc elle est apte à générer des écoulements diluviaux en périodes des précipitations orageuses.

\section{Index de Forme (F)}

L'Index de Forme d'un bassin versant est la relation existant entre la largeur (B) et la longueur (L) du bassin. Si un bassin versant a une valeur de $\mathrm{F}$ proche de zéro (0), cela signifie qu'il a une forme allongée et par conséquent plus il se produit d'orages à l'intérieur de celui-ci. Si la valeur de F est proche de 1, la possibilité pour qu'il y ait une concentration de fortes pluies, génératrices d'écoulements torrentiels, est moindre. D'où la formule :

$$
F=\frac{B}{L}
$$

Pour le bassin versant de l'Oued Beht, on a la largeur B $=51,43 \mathrm{~km}$ et la longueur $\mathrm{L}=121,43 \mathrm{~km}$. Ce qui donne un Index de Forme $\mathbf{F}=\mathbf{0 , 4 2}$ proche de zéro ; cela signifie que le bassin versant a une forme allongée et par conséquent il est favorable à une naissance d'écoulements diluviens en périodes des pluies orageuses.

\section{Caractéristiques topographiques (Relief)}

L'influence du relief sur l'écoulement est encore plus évidente et se conçoit aisément, car de nombreux paramètres hydrométéorologiques varient avec l'altitude (précipitations, températures, etc.) et la morphologie du bassin versant. En outre, la pente influe sur la vitesse d'écoulement, les styles fluviaux que prennent les cours d'eau et l'évolution spatiale de l'importance des processus sédimentaires fluviatiles. 
Sur la carte, le relief est indiqué par les courbes de niveau en joignant les points d'égale altitude. Il est nécessaire de répartir le bassin versant en tranches d'altitude à fin de pouvoir dégager les différents paramètres du relief. La répartition des altitudes a été déterminée à partir d'un MNT de $30 * 30 \mathrm{~m}$ de résolution. Les résultats, obtenus à l'aide des logiciels SIG, ont permis d'établir la carte et la courbe hypsométriques.

\section{Modèle Numérique de Terrain (MNT)}

Le Modèle Numérique de Terrain (MNT) est un terme générique couvrant les données numériques de la topographie (altimétrie pour les secteurs émergés ou bathymétrie pour les secteurs submergés) d'une zone terrestre ou d'une planète tellurique, dans toutes les formes adaptées à son utilisation par un calculateur numérique (ordinateur), ainsi que par toutes les méthodologies possibles (Maune et al., 2001). C'est le synonyme d'un MNE du sol nu. Il couvre les modèles d'altitude et autres éléments géographiques. Il peut également inclure des produits dérivés comme la pente, l'azimut, entre autres (Li et al., 2004). Il existe trois sources possibles de données pour la construction d'un MNT : les mesures directes sur le terrain (GPS) ; les mesures directes à distance (LiDAR); et les mesures indirectes à distance comme l'application de la stéréophotogrammétrie à partir d'images stéréoscopiques aériennes ou de satellites. Ces dernières ont été jusqu'à aujourd'hui le moyen le plus utilisé pour la production d'information sur le relief.

Le Modèle Numérique de Terrain (MNT) est une représentation numérique du relief donc des valeurs d'altitude d'une zone donnée. Il permet d'avoir une représentation en 3 dimensions (3D) du bassin versant. Il est établi à partir des courbes de niveau numérisées du bassin. Les altitudes sont calculées aux points d'une grille dont la taille d'une maille élémentaire détermine le pas du modèle. On peut en dériver des indications sur différents paramètres: altitude moyenne, direction de drainage, pente moyenne, exposition, formes de la surface topographique etc.

Si l'on représente uniquement l'altitude du sol nu, on parle de MNT. Si l'on prend en compte les hauteurs de tous les objets placés sur celui-ci comme les bâtiments et la végétation, ce que l'on appelle le « sursol», on parle alors de Modèle Numérique d'Altitude (MNA). Un MNT peut prendre la forme de fichiers vecteurs (points, polylignes où chaque entité porte l'information altimétrique), d'un fichier raster (où chaque pixel ou cellule porte l'information altimétrique), d'un Réseau de Triangles Irréguliers (TIN).

Morphologiquement, le MNT du BV de l'Oued Beht présente une topographie avec une dénivelée importante entre 65 et $2187 \mathrm{~m}$. Il montre dans ses parties amont, situées dans le Causse moyen atlasique d'âge jurassique 
dans la région d'Azrou, des zones montagneuses dont l'altitude atteint $2187 \mathrm{~m}$. Vers le NW, les altitudes diminuent progressivement dans le Maroc Central caractérisé par une multitude de collines offrant un relief aéré d'âge paléozoïque (Fig.2).

Ensuite, le BV se rétrécit et change de direction. En effet, il s'élargit de nouveau et se développe vers le Nord dans les Rides sud-rifaines avec une diminution progressive de l'altitude jusqu'à $65 \mathrm{~m}$ dans les régions planes avoisinantes du barrage El Kansera, situé à l'aval du BV dans la région de Khémisset (Fig. 2).

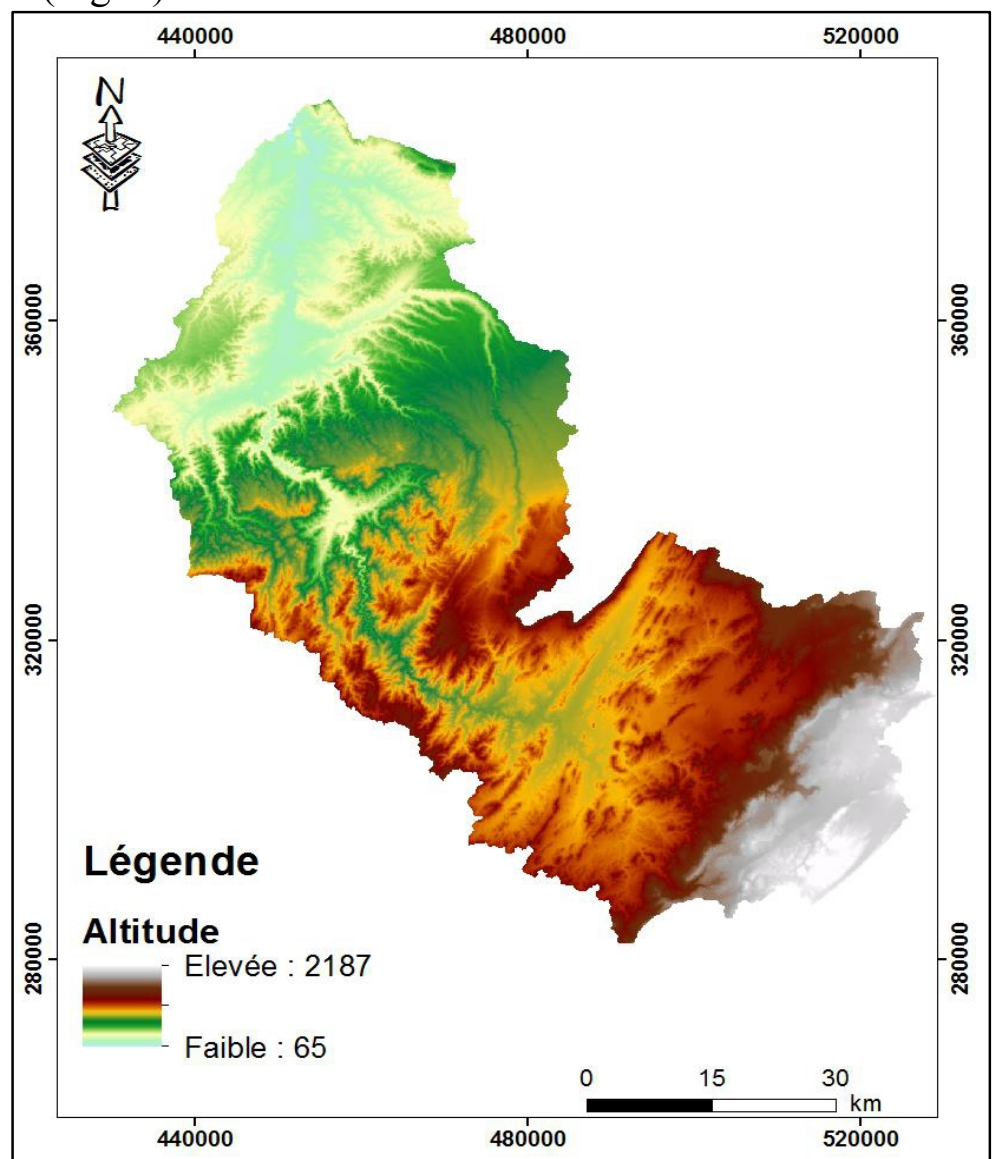

Figure 2. Modèle Numérique de Terrain (MNT) du Bassin versant de l'Oued Beht.

\section{Carte hypsométrique}

Le modèle numérique de terrain (MNT) permet de réaliser une analyse spatiale représentative car il reflète des informations relatives à la structure morphologique sur l'ensemble du bassin versant (Zhou, 2009). Il donne aussi des prévisions sur la nature et l'intensité des précipitations.

Les surfaces comprises entre les courbes de niveau de la carte hypsométrique sont représentées sous formes de classes de couleurs 
différentes (Fig. 3), en partant de la classe de haute altitude dont le point culminant se tient à $2187 \mathrm{~m}$ jusqu'à celle de basse altitude dont le point le plus bas présente une altitude égale à $65 \mathrm{~m}$.

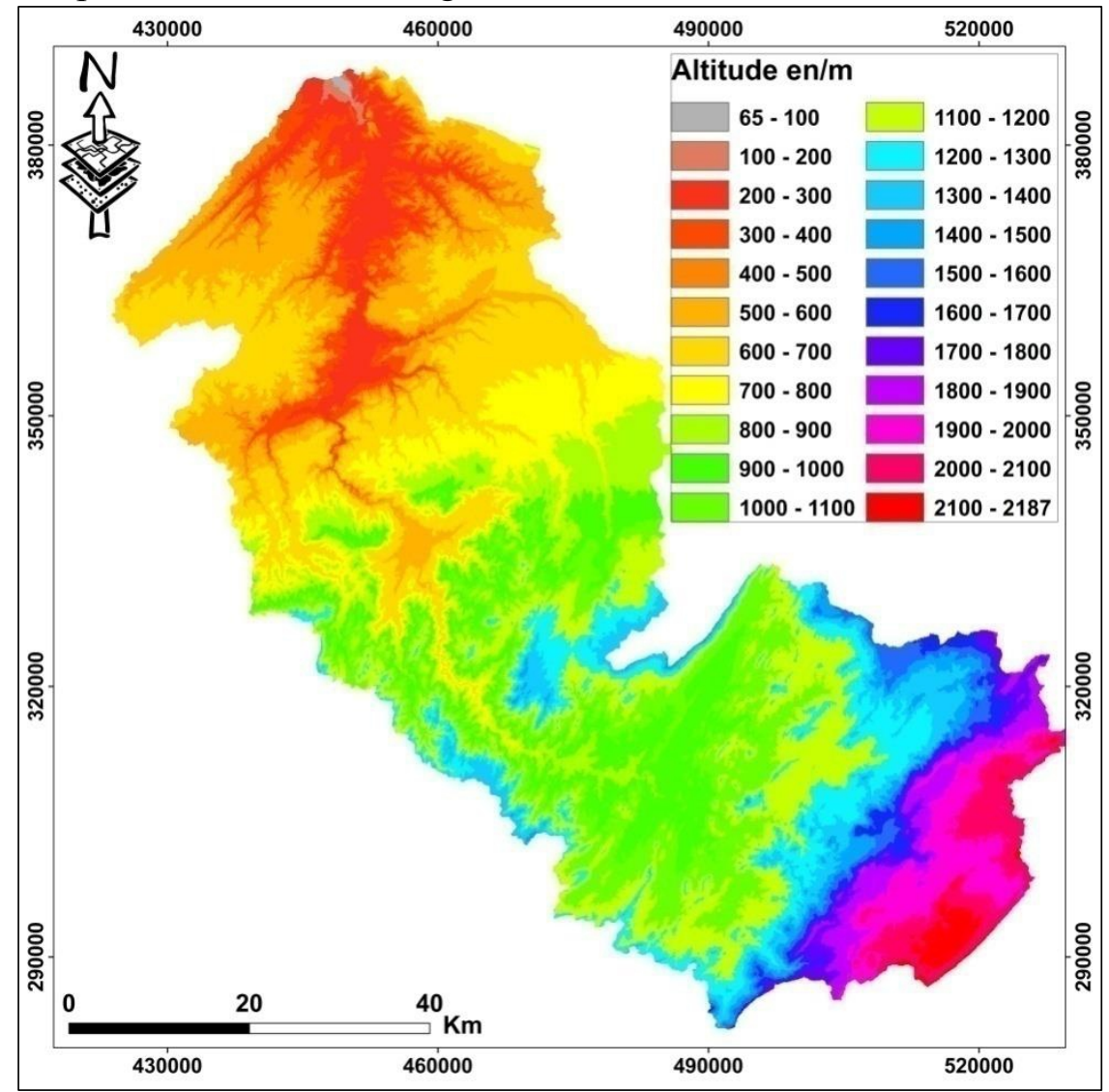

Figure 3. Carte hypsométrique du bassin versant de Beht en amont du barrage El Kansera.

La carte hypsométrique présente des tranches d'altitudes équidistantes de $100 \mathrm{~m}$. Ces tranches, généralement orientées NE-SW, témoignent de la structure du bassin versant en plateaux étagés. Les zones de hautes altitudes figurent dans le SE du bassin et les zones de basses altitudes se trouvent au NW et aux alentours du barrage El Kansera (Fig. 3).

\section{Courbe hypsométrique}

Le relief d'un bassin est souvent caractérisé par la courbe de sa répartition hypsométrique. La courbe hypsométrique fournit une vue synthétique de la pente du bassin versant et représente la répartition de la surface du bassin versant en fonction de son altitude. Cette courbe, qui porte en abscisse le pourcentage de surface (surface cumulée) du bassin qui se trouve au dessus de l'altitude représentée en ordonnée, reflète aussi son état 
d'équilibre dynamique potentiel. Elle demeure un outil pratique pour comparer plusieurs bassins versants entre eux ou les diverses sections d'un seul bassin. Elle peut en outre servir à la détermination de la pluie moyenne sur un bassin versant et donner des indications quant au comportement hydrologique et hydraulique de celui-ci et de son système de drainage.

Cette courbe, qui permet de juger de l'âge et du degré d'érosion des bassins versants, constitue aussi un outil de comparaison entre des sous bassins versants. En effet, la forme de la courbe hypsométrique est caractéristique de l'état de maturité du relief (jeune, en équilibre et vieux) ainsi que de sa capacité érosive (Musy, 2005). Selon Strahler (1952), l'allure de la courbe hypsométrique caractérise le cycle d'érosion du relief actuel étudié (Fig. 4). En effet, une courbe convexe est caractéristique d'un relief accusé et jeune où l'érosion est encore intense, tandis qu'une forme concave reflète un vieux relief non rajeuni par les soulèvements tectoniques et où l'érosion est devenue faible et stable (Bannister, 1980). L'interprétation de l'aspect de la courbe hypsométrique est donnée en fonction du degré d'érosion associé à l'âge du bassin (Fig. 4) :

* Les bassins plus jeunes montrent une superficie faible par rapport au changement d'altitude initiale, ce qui est caractéristique des bassins abrupts.

Les vieux bassins présentent de larges topographies pratiquement planes où l'altitude varie très peu.

Les bassins, correspondant à la courbe du milieu, sont des bassins proches de l'état dit d'équilibre ou « mature ».

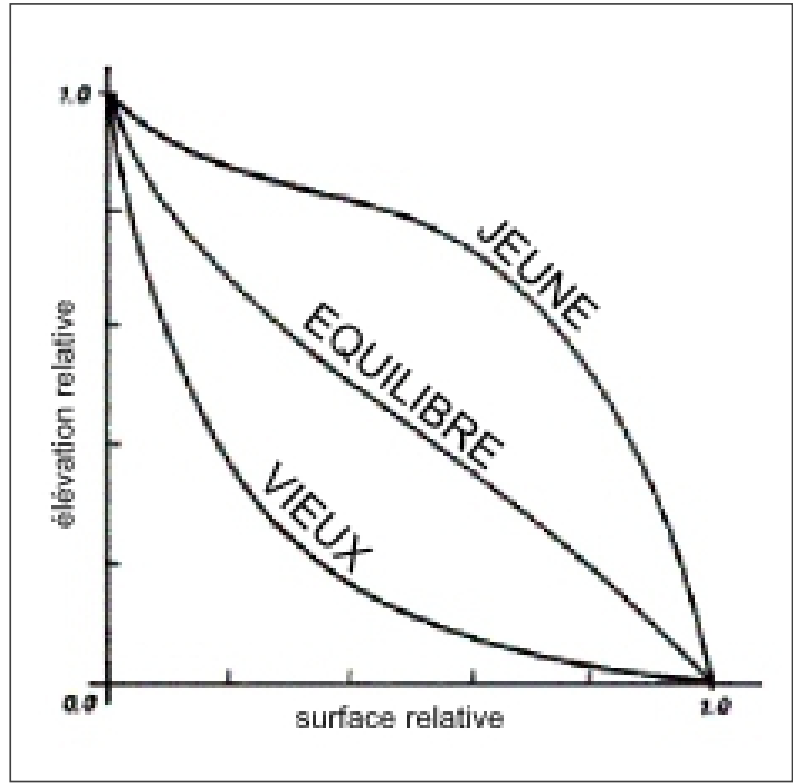

Fig. 4. Etat de maturité du relief des BV. 
La courbe hypsométrique du bassin versant de l'Oued Beht a été tracée à partir des élévations par tranches d'altitudes (en mètre) et des surfaces relatives calculées $\left(\mathrm{en}^{2} \mathrm{~km}^{2}\right.$ ). Elle montre une légère concavité vers le bas, avec une pente assez élevée vers les hautes altitudes. Vers les basses altitudes, la pente devient faible (Fig. 5 et Tab. 1). Ces deux zones sont séparées par une zone d'inflexion dont le point culminant coïncide avec l'altitude médiane de $900 \mathrm{~m}$. Cette dernière correspond de point de vue structural à un dôme qui sépare deux dépressions.

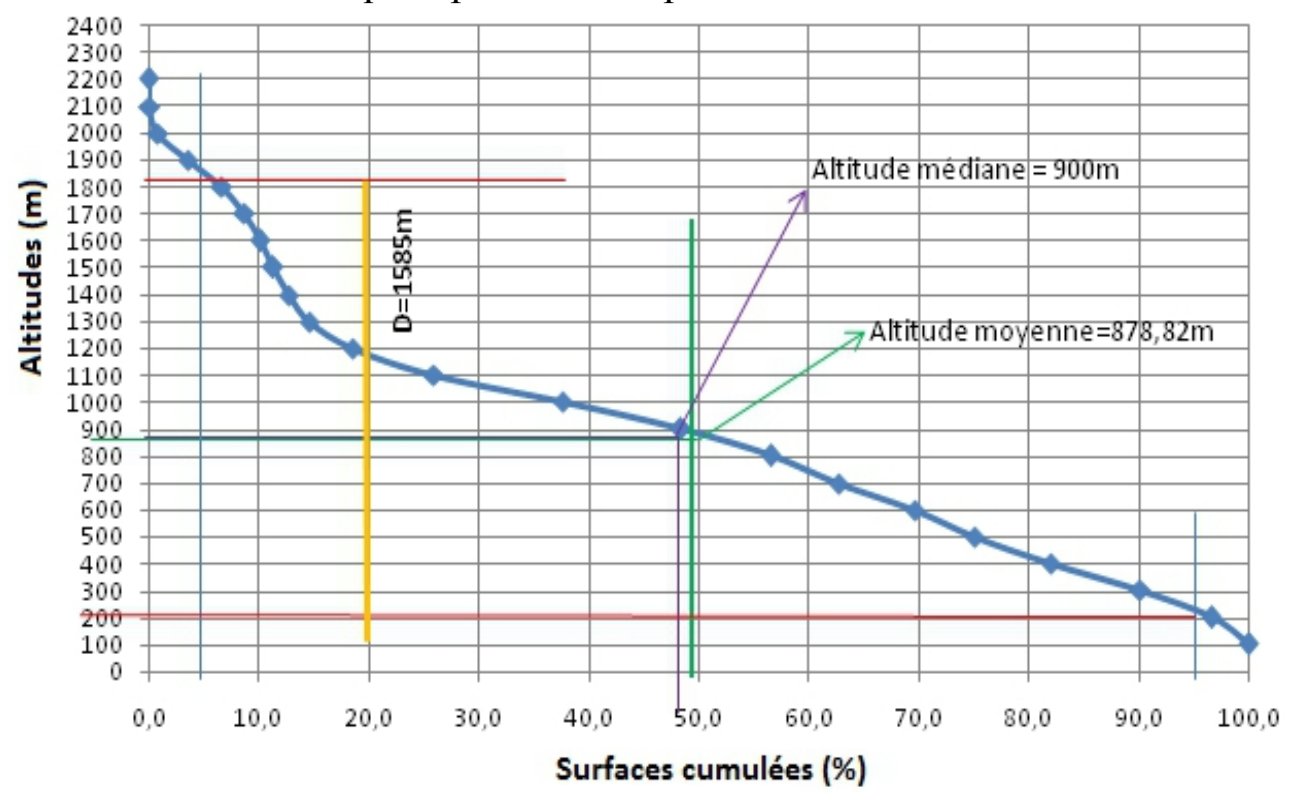

Figure 5. Courbe hypsométrique du bassin versant de l'Oued Beht avec Dénivelée (D), altitude moyenne et altitude médiane.

Les aires situées au dessous du profil d'équilibre, matérialisé par la droite passant par l'abscisse $100 \%$ des surfaces cumulées et l'ordonnée $2200 \mathrm{~m}$ d'altitude, sont supérieures à celles se trouvant en dessus. La courbe hypsométrique du bassin versant de l'Oued Beht est celle d'un bassin versant qui a déjà dépassé l'état de maturité et d'équilibre (Fig. 4 et 5) et a atteint par conséquent un stade relativement développé d'érosion. Nous pouvons d'ores et déjà avancer qu'au niveau de ce bassin versant, le pouvoir érosif lié à l'eau est très développé. Toutefois, le passage de ce stade actuel vers le stade vieux nécessite une érosion qui doit se faire au niveau des parties supérieure et moyenne du profil longitudinal du Moyen Beht et un alluvionnement dans sa partie inférieure. Cette évolution entrainerait un envasement du barrage El Kansera situé en aval du bassin versant en l'absence d'aménagement adéquat. 
La forte pente du profil longitudinal du cours d'eau supérieur ou amont de l'Oued Beht est liée à deux raisons géologiques :

+ La lithologie du substrat, relativement plus dure que celle du substrat d'aval, qui est composée de formations schisto-gréso-quartizitiques du socle paléozoïque que coiffe une couverture mésozoïque constituée d'argiles et de silts rouges à intercalation de basaltes triasiques qu'arment des terrains dures calcaro-dolomitiques du Jurassique.

+ L'exhaussement tectonique de cette zone qui correspond à un compartiment soulevé par un réseau de failles composé principalement de deux familles : une famille de direction SW-NE et une autre qui lui est orthogonale et de direction SE-NW. La première famille, de direction SWNE aurait joué un rôle beaucoup plus important dans la structuration et le compartimentage de la partie amont du bassin de l'Oued Beht en compartiments de plus en plus affaissés vers le NW. Les expressions géomorphologique et topographique de ces compartiments tectoniques sont des unités topographiques plus au moins planes correspondant à des plateaux. Ces unités se présentent sur la carte hypsométrique (Fig.3) sous forme de bandes d'altitudes : 2100-2200, 2000-2100, 1900-2000, 1900$1800 \mathrm{~m}$ etc. Les ruptures de pente qui séparent ces unités topographiques matérialisent le passage de ces failles ou accidents tectoniques. Soulignons que cette tectonique, qui est responsable de ce compartimentage en blocs étagés, s'exprimant dans la topographie sous forme de plateaux dans la partie amont du bassin, l'est aussi dans la totalité du bassin versant. En effet celui-ci est formé d'une succession de plateaux étagés de plus en plus bas, qu'on peut classer en hauts, moyens et bas plateaux et qui passent vers l'aval à des plaines dans la zone d'installation du barrage El Kensra. Ces différentes unités topographiques ou géomorphologiques ne sont en fait que l'expression d'un compartimentage tectonique en blocs étagés ayant été plus ou moins modifiés et modelés par les processus de géomorphogenèse surtout quaternaires qui continuent à se produire actuellement.

Tableau 1. Répartition par tranches d'altitudes de la superficie $\left(\mathrm{Km}^{2}\right)$ du bassin versant de l'Oued Beht.

\begin{tabular}{|c|c|c|c|c|}
\hline $\begin{array}{c}\text { Elévation } \\
\text { courbe de } \\
\text { niveau [m] }\end{array}$ & $\begin{array}{c}\text { Surface entre les } \\
\text { courbes }\left(\mathrm{km}^{2}\right)\end{array}$ & $\begin{array}{c}\text { Pourcentage } \\
\text { du total }(\%)\end{array}$ & Surface cumulée (\%) & $\begin{array}{c}\text { Pourcentage au } \\
\text { dessus de la } \\
\text { limite inférieure }\end{array}$ \\
\hline $65-100$ & 1,55884 & 0,03 & 0,03 & 100,0 \\
\hline $100-200$ & 154,6113 & 3,36 & 3,39 & 96,61 \\
\hline $200-300$ & 299,8771 & 6,51 & 9,90 & 90,1 \\
\hline $300-400$ & 375,9138 & 8,17 & 18,07 & 81,9 \\
\hline $400-500$ & 314,2079 & 6,83 & 24,89 & 75,1 \\
\hline $500-600$ & 251,3422 & 5,46 & 30,35 & 69,6 \\
\hline $600-700$ & 319,5094 & 6,94 & 37,29 & 62,7 \\
\hline $700-800$ & 286,9018 & 6,23 & 43,52 & 56,5 \\
\hline
\end{tabular}




\begin{tabular}{|c|c|c|c|c|}
\hline $800-900$ & 374,2571 & 8,13 & 51,65 & 48,3 \\
\hline $900-1000$ & 494,107 & 10,73 & 62,39 & 37,6 \\
\hline $1000-1100$ & 538,4323 & 11,70 & 74,08 & 25,92 \\
\hline $1100-1200$ & 339,2397 & 7,37 & 81,45 & 18,55 \\
\hline $1200-1300$ & 183,8 & 3,99 & 85,44 & 14,56 \\
\hline $1300-1400$ & 81,96334 & 1,78 & 87,22 & 12,78 \\
\hline $1400-1500$ & 67,40664 & 1,46 & 88,69 & 11,31 \\
\hline $1500-1600$ & 53,58041 & 1,16 & 89,85 & 10,15 \\
\hline $1600-1700$ & 66,72135 & 1,45 & 91,30 & 8,70 \\
\hline $1700-1800$ & 94,56208 & 2,05 & 93,35 & 6,65 \\
\hline $1800-1900$ & 143,8726 & 3,13 & 96,48 & 3,52 \\
\hline $1900-2000$ & 123,8562 & 2,69 & 99,17 & 0,83 \\
\hline $2000-2100$ & 34,85927 & 0,76 & 99,93 & 0,07 \\
\hline $2100-2187$ & 3,185455 & 0,07 & 100,00 & 0,00 \\
\hline Total & & 100,00 & & \\
\hline
\end{tabular}

En résumé, l'analyse de la carte et de la courbe hypsométrique confirme qu'on est en présence d'un bassin où les différences d'altitudes sont très marquées avec différenciation d'une structure en plateaux étagés de plus en plus affaissés en allant vers le NW du bassin. Ces contrastes altimétriques, liés à des conditions morpho-litho-structurales différentes le long du bassin, favorisent l'écoulement des eaux de ruissellement à l'intérieur du bassin qui dispose d'un rare couvert forestier. Ces conditions topo-hydro-biologiques vont permettre des pertes en terres plus grandes en provenance des sols et des formations géologiques surtout les moins compétentes composées de schistes, d'argiles silteuses et de marnes sableuses.

\section{Rectangle équivalent}

Le rectangle équivalent, ou rectangle de Gravelius, se définit comme le rectangle qui a la même surface et le même périmètre, le même indice de compacité et la même distribution hypsométrique à conditions climatiques similaires, une même distribution des formations lithologiques et pédologiques, de la végétation et même densité de drainage que le bassin versant. C'est une transformation purement géométrique dans laquelle les droites parallèles aux largeurs du rectangle et l'exutoire sont le petit coté ou largeur du rectangle (Laborde, 2009). Les courbes de niveau y sont parallèles et sont tracées en respectant les répartitions hypsométriques. La répartition du bassin versant par tranches d'altitude, dans un rectangle équivalent, donne une importance capitale pour les études hydrologiques du fait que la plupart des facteurs météorologiques et hydrologiques sont influencés par l'altitude Cette notion a été introduite pour pouvoir comparer des bassins versants 
entre eux du point de vue de l'influence de leurs caractéristiques géométriques sur l'écoulement. Il faut noter que moins un bassin versant est a11ongé mieux il est drainé.

Soit Leq et leq, la longueur et la largeur du rectangle (exprimées en $\mathrm{km}$ ), $\pi=3,14$, $\mathrm{P}$, le périmètre en $\mathrm{km}$ et $\mathrm{A}$, l'aire du bassin versant, exprimée en $\mathrm{km}^{2}$. Sa longueur et sa largeur sont données à partir de son indice de compacité $\mathrm{K}_{\mathrm{G}}$ et de sa superficie A, par les expressions :

$$
\begin{aligned}
& \text { Leq }=\frac{1}{2} \cdot \text { KG } \cdot \sqrt{\pi \cdot A} \cdot\left(1+\sqrt{1-\left(\frac{2}{\sqrt{\pi} \cdot \mathrm{KG}}\right)^{2}}\right. \\
& \text { leq }=\frac{1}{2} \cdot \text { KG. } \sqrt{\pi \cdot A} \cdot\left(1-\sqrt{1-\left(\frac{2}{\sqrt{\pi} \cdot \mathrm{KG}}\right)^{2}}\right.
\end{aligned}
$$

Pour le bassin versant de l'Oued Beht, Leq $=\mathbf{1 5 8 , 7 4} \mathbf{~ k m}$ et leq=29 km. Ces dimensions traduisent que la longueur est 5,47 fois plus grande que la largeur. Elles permettent également de dessiner le rectangle équivalent de la zone d'étude (Fig. 6) en se basant sur les données de la carte et de la courbe hypsométriques (Fig. 3 et 5 et Tab. 1).

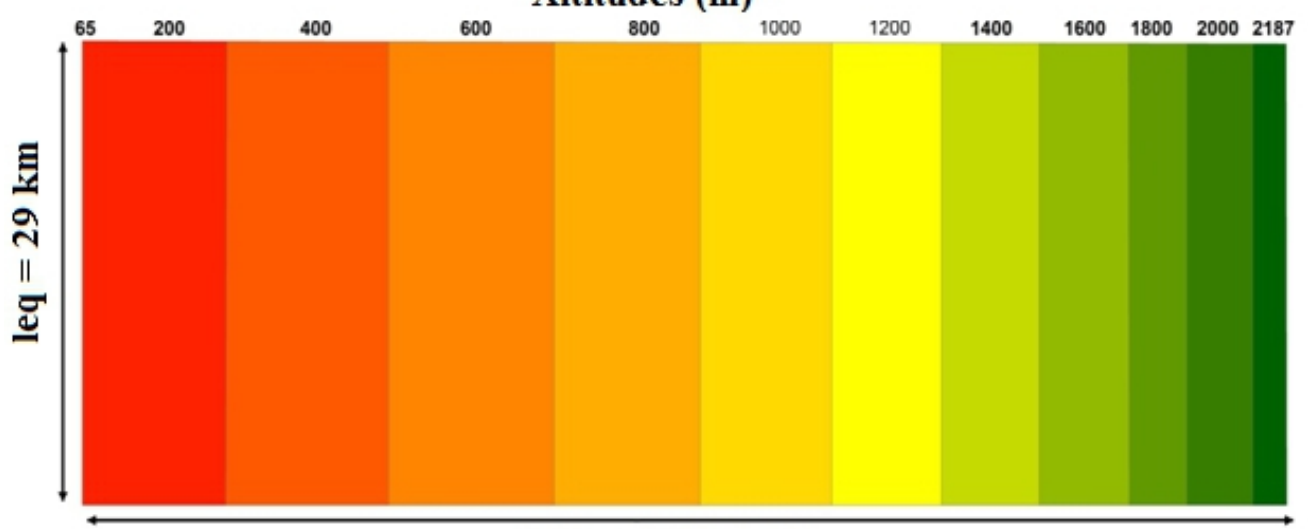

$$
\text { Leq }=158,74 \mathrm{~km}
$$

Figure 6. Rectangle équivalent avec tranches d'altitudes (m) du basin versant de l'Oued Beht.

Les courbes de niveau ou les droites parallèles sont assez serrées en amont du bassin versant témoignant d'une pente importante dans cette partie du bassin et d'un relief fort, montré par les altitudes de ces droites. Elles deviennent progressivement plus espacées en allant vers l'exutoire du bassin en parallèle avec la diminution de la pente et le passage vers la plaine (Fig. $6)$. 


\section{Altitudes caractéristiques}

\section{Altitudes extrêmes}

Les principales altitudes extrêmes du bassin versant de l'oued Beht, extraites à partir du MNT et de la courbe hypsométrique (Fig. 7 et 8), sont les suivantes :

- L'altitude minimale est : Hmin $=65 \mathrm{~m}$;

- L'altitude maximale est : Hmax = $2187 \mathrm{~m}$;

- La hauteur à $5 \%$ de la surface totale est : $\mathrm{H}_{5 \%}=1800 \mathrm{~m}$;

- La hauteur à $95 \%$ de la surface totale est : $\mathrm{H}_{95 \%}=215 \mathrm{~m}$;

Les altitudes maximales et minimales sont obtenues directement à partir des cartes topographiques ou bien du MNT à l'aide des logiciels du SIG comme c'est le cas dans le présent travail (Fig. 4 et 5). L'altitude maximale représente le point le plus élevé du bassin tandis que l'altitude minimale considère le point le plus bas, généralement à l'exutoire. Ces deux données deviennent surtout importantes lors du développement de certaines relations faisant intervenir des variables climatologiques telles que la température, la précipitation et le couvert neigeux. Elles déterminent l'amplitude altimétrique du bassin versant et interviennent aussi dans le calcul de la pente.

\section{Altitude moyenne}

L'altitude moyenne est peu représentative de la réalité. Toutefois, elle est parfois utilisée dans l'évaluation de certains paramètres hydrométéorologiques ou dans la mise en œuvre de modèles hydrologiques. Elle se déduit directement de la courbe hypsométrique ou de la lecture d'une carte topographique. Elle est définie par:

Avec :

$$
\text { Hmoy }=\sum_{1}^{n} \frac{\mathrm{Ai} \cdot \mathrm{hi}}{\mathrm{A}}
$$

$\mathrm{H}_{\text {moy }}$ : altitude moyenne du bassin [m];

Ai : aire comprise entre deux courbes de niveau $\left[\mathrm{km}^{2}\right]$;

hi : altitude moyenne entre deux courbes de niveau [m] ;

A : superficie totale du bassin versant $\left[\mathrm{km}^{2}\right]$.

L'altitude moyenne du bassin versant de l'Oued Beht est Hmoy $=878,82 \mathrm{~m}$ calculée manuellement d'après la formule ci-dessus et le tableau 2. 
Tab.2 : Calcule de l'altitude moyenne du BV de l'oued Beht.

\begin{tabular}{|c|c|c|}
\hline $\mathrm{Ai}$ & hi & Ai.hi \\
\hline 13,56 & 90,7 & 1229,89 \\
\hline 154,61 & 150 & 23191,69 \\
\hline 300,88 & 250 & 75219,27 \\
\hline 375,91 & 350 & 131569,83 \\
\hline 315,21 & 450 & 141843,53 \\
\hline 252,34 & 550 & 138788,20 \\
\hline 319,51 & 515 & 164547,35 \\
\hline 286,90 & 750 & 215176,35 \\
\hline 374,26 & 850 & 318118,51 \\
\hline 494,11 & 950 & 469401,64 \\
\hline 540,43 & 1050 & 567453,86 \\
\hline 339,24 & 1150 & 390125,60 \\
\hline 183,80 & 1250 & 229750,00 \\
\hline 81,96 & 1350 & 110650,50 \\
\hline 69,41 & 1450 & 100639,62 \\
\hline 53,58 & 1550 & 83049,63 \\
\hline 66,72 & 1650 & 110090,23 \\
\hline 94,56 & 1750 & 165483,63 \\
\hline 143,87 & 1850 & 266164,34 \\
\hline 123,86 & 1950 & 241519,61 \\
\hline 35,86 & 2050 & 73511,50 \\
\hline 13,19 & 2150 & 28348,73 \\
\hline & $\sum(\mathrm{Ai} . \mathrm{hi})$ & 4045873,52 \\
\hline
\end{tabular}

\section{Altitude médiane}

L'altitude médiane correspond à l'altitude lue au point d'abscisse $50 \%$ de la surface totale du bassin, sur la courbe hypsométrique. Cette grandeur se rapproche de l'altitude moyenne, dans le cas où la courbe hypsométrique, du bassin concerné, présente une pente régulière. L'altitude médiane dans le bassin versant de l'Oued Beht est égale à $\mathrm{H}_{\mathrm{med}}=900 \mathrm{~m}$ (Fig. 5).

Ainsi, l'altitude moyenne $($ Hmoy $=878,82 \mathrm{~m})$ est inférieure à l'altitude médiane $\left(\mathrm{H}_{\mathrm{med}}=900 \mathrm{~m}\right)$ avec un écart de $21,18 \mathrm{~m}$. Donc la pente moyenne du bassin versant est irrégulière. Elle est trop forte en amont du bassin, où prédominent les hauts reliefs, et s'adoucit en descendant vers l'aval où dominent des bas plateaux et des plaines.

\section{Pente moyenne du bassin versant}

La pente moyenne $\left(I_{m}\right)$ d'un bassin versant est un paramètre qui a un rapport important et complexe avec l'infiltration, le ruissellement superficiel et la saturation du sol en eau. Elle contrôle le temps de ruissellement et de 
concentration des eaux pluviales dans les artères de drainage. Plusieurs méthodes ont été développées pour estimer la pente moyenne d'un bassin versant. Elles se basent toutes sur la lecture d'une carte topographique réelle ou approximative ou bien à partir d'un MNT en utilisant le SIG comme c'est le cas dans le présent travail.

- La méthode proposée par Cartier et Leclerc (1964) consiste à calculer la moyenne pondérée des pentes de toutes les surfaces élémentaires comprises entre deux altitudes données. Une valeur approchée de la pente moyenne est alors donnée par la relation suivante :

Où:

$$
\mathrm{I}_{\mathrm{m}}=100 . \frac{\mathrm{Eq} * \mathrm{Lcn}}{\mathrm{A}}
$$

Lcn : longueur totale des courbes de niveau $[\mathrm{km}]=6158,37 \mathrm{~km}$. Elle peut être mesurée par les logiciels SIG comme ArcGIS ou Global Mapper. $\mathrm{Eq}$ : équidistance entre deux courbes de niveau $[\mathrm{km}]=100 \mathrm{~m}=0,1 \mathrm{~km}$.

A : surface du bassin versant $\left[\mathrm{km}^{2}\right]=4603,77 \mathrm{~km}^{2}$.

$\mathrm{I}_{\mathrm{m}}$ : pente moyenne $[\mathrm{m} / \mathrm{km}$ ou $\%]=100 *(0,1 * 6158,37) / 4603,77=\mathbf{1 3 , 3 8 \%}$.

La pente moyenne du bassin versant de l'Oued Beht $(\mathrm{Im}=13,38 \%)$ est une pente douce à modérée, qui impliquera une durée moyenne de concentration des eaux de ruissellement dans les affluents. L'application de la formule utilisée pour la pente moyenne donne de bons résultats, dans le cas d'un relief modéré et pour des courbes de niveau simples et uniformément espacées. Dans les autres cas, il convient de styliser les courbes de niveau pour que leur longueur totale ait un sens réel vis-à-vis de la pente.

- Une façon simple pour évaluer la pente moyenne $\left(I_{m}\right)$ selon Llamas (1993) est de calculer la pente moyenne $\mathrm{Im}$ avec :

$$
\mathrm{I}_{\mathrm{m}}=\frac{2 \mathrm{DT}}{\mathrm{P}}=\frac{2 * 2122}{421,43}=10,07
$$

Avec $\mathrm{D}_{\mathrm{T}}=$ dénivellation totale entre l'embouchure et le point le plus haut du bassin versant $=H \max -\mathrm{Hmin}=2122 \mathrm{~m}$.

$\mathrm{P}=$ périmètre du bassin versant $=421,43 \mathrm{~km}$. Donc, la pente moyenne $\mathbf{I m}=$ $10,07 \%$.

Cependant, cette formule si elle présente l'intérêt d'être rapide a l'inconvénient de lisser les irrégularités d'un relief profondément érodé comme celui de l'Oued Beht. II vaut donc mieux prendre la première formule.

- La méthode la plus simple pour calculer la pente moyenne $\left(\mathrm{I}_{\mathrm{m}}\right)$ consiste à mesurer la dénivelée totale du bassin avec la longueur du rectangle équivalent.

$$
\mathrm{I}_{\mathrm{m}}=\frac{\mathrm{DT}}{\mathrm{Leq}}=\frac{2122}{158,74}=13,37 \%
$$


Avec $\mathrm{D}_{\mathrm{T}}=$ dénivellation totale entre l'embouchure et le point le plus haut $\mathrm{du}$ bassin versant $=\mathrm{Hmax}-\mathrm{Hmin}=2122 \mathrm{~m}$. Leq $=$ longueur du rectangle équivalent $=158,74 \mathrm{~km}$.

Donc, la pente moyenne $\mathbf{I m}=\mathbf{1 3 , 3 7} \%$. Il s'agit donc d'une pente modérée qui pourrait causer un grand risque d'érosion au niveau du bassin versant (Motoc, 1975).

On note une nette différence entre la formule de Cartier et Leclerc (1964) et celle de Llamas (1993) qui montre que l'érosion intense a raviné le paysage créant un aspect fractal qui ne peut etre pris en compte que par l'allure des courbes de niveau. La pente moyenne de 13,38 \% est modérée et expliquerait la réponse qui devrait être relativement lente du bassin versant aux pluies. Elle traduit bien non seulement la pente relative du bassin, mais aussi l'existence de ravines profondes disséminées sur le relief et siège de l'écoulement des principaux bras et affluents. L'indice proposé par Llamas (1993) donne une pente lissée qui correspond à la pente moyenne des cours d'eau (ORE, 1998).

\section{Carte des pentes}

La pente topographique est un paramètre capital dans tout travail d'aménagement ; elle conditionne le drainage (Abbassi, 2004). En effet, elle affecte d'une façon directe par son intensité les phénomènes d'infiltration, de ruissellement et d'érosion, sa forme, et la longueur sur laquelle elle agit.

La carte des pentes du bassin versant de l'Oued Beht a été établie à partir du MNT de la région étudiée (Fig. 7). Elle montre que les pentes les plus abruptes, très fortes à fortes, se concentrent dans la partie SW dans le Maroc central, où prédominent les terrains paléozoïques, et dans le $\mathrm{SE}$ du bassin versant dans le causse du Moyen Atlas. 


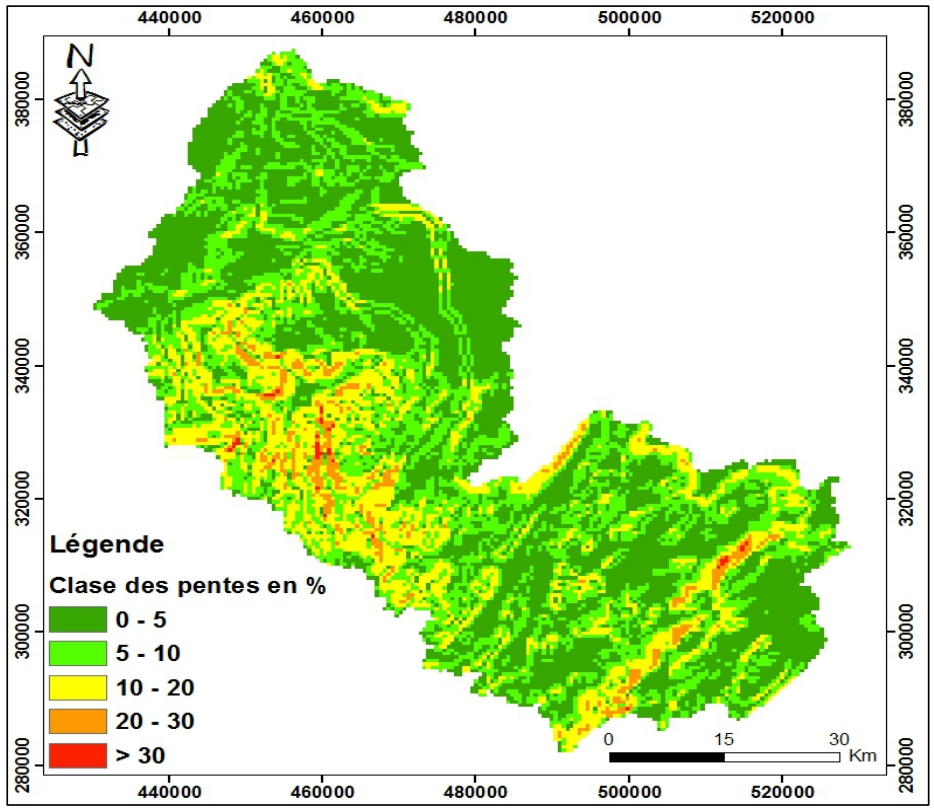

Figure 7. Carte des pentes du bassin versant de l'Oued Beht.

Les pentes faibles à très faibles se développent dans les plaines plioquaternaires dans la partie aval du bassin versant juste avant le barrage El Kansera (Fig. 7).

La carte des pentes montre que plus de $88 \%$ de la topographie du bassin présente une pente inférieure à $5 \%$ et le reste de cette topographie, soit $12 \%$ de la superficie du bassin versant, présente une pente qui varie entre 5 et $>20 \%$ et la classe des pentes les plus fortes (>20\%) n'en couvre que 6,5\% (Tab. 3 et Fig. 7).

Ces valeurs de pente sont à mettre en relation avec la structure géologique et surtout la lithologie des terrains qui déterminent leur comportement vis à vis de l'érosion. L'observation et l'analyse de la carte des pentes (Fig.7) confirment les données de la carte hypsométrique et montrent que la topographie du bassin est formée en amont de plateaux étagés du SE vers le NW (Beaudet, 1996) qui passent vers l'aval à des plaines. Toutefois parmi ces unités topographiques, il y en a qui présentent des topographies accidentées liées à des entités topographiques de pente accusée. Ces topographies sont localisées dans la partie amont du SE du bassin et au NW de sa partie médiane. Les fortes valeurs de pente des versants sont liées à deux causes géologiques : la lithologie et la tectonique. La cause lithologique est la plus importante et due à l'existence de formations lithologiques de dureté ou de compétence différentes.

Dans la partie SE du bassin, ces formations sont composées de schistes, de grès et quartzites d'âge paléozoïque. Ces formations du socle 
présentent un relief structural de type appalachien (Beaudet, 1996). Dans ce relief, les schistes, tendres et facilement érodables, forment des sillons où les pentes sont faibles alors que les grès et les quartzites, dures et très résistants à l'érosion, forment des crêts où les valeurs de pente sont beaucoup plus fortes.

Dans le NW de la partie médiane du bassin versant, dans le Sillon sud-rifain, affleurent également des roches tendres et des roches dures. Les roches tendres sont formées par des argiles et des silts rouges gyspsosalifères d'âge triasique et des marnes sableuses grises d'âge miocène supérieur alors que les roches dures sont constituées de grès jaunâtres d'âge miocène supérieur. Les pentes les plus faibles se répartissent dans les zones d'affleurement des roches tendres alors que les pentes relativement fortes sont rattachées aux affleurements des grès.

La limite entre la zone amont du bassin, constituée par le Plateau central et le Causse moyen atlasique, et la partie médiane, formée par le Sillon sud rifain, est marquée par une accentuation de la pente. Cette accentuation correspondrait au talus d'un accident tectonique important de direction SW-NE dont le dénivelé a été atténué par l'érosion.

Les cartes des pentes révèlent la présence de ruptures et de variations morphologiques le long des pentes (Collin, 2009). Ces ruptures de pente et variations morphologiques sont liées à des variations de lithologie, de structure géologique et surtout à une existence de failles importantes. Les fortes pentes accentuent le phénomène d'érosion grâce à l'instabilité des terrains, surtout lorsque les versants sont dénudés ou présentent un faible couvert végétal. La mobilité des masses d'air, causée par le dénivelé d'altitude, donne naissance aux courants d'air qui se mobilisent vers les plaines (Benyassine et Dekayir, 2013). Ces courants jouent un rôle important dans le transport éolien d'éléments détritiques érodés sur la totalité du bassin versant, surtout lorsque le climat est aride et le couvert forestier est absent ou très faible.

Tableau 3. Classification des pentes du bassin versant de l'oued Beht en amont du barrage El Kansera.

\begin{tabular}{|c|c|c|c|c|}
\hline & Pente & $\begin{array}{c}\text { Valeur de la } \\
\text { pente en \% }\end{array}$ & $\begin{array}{c}\text { Superficie en } \\
\mathrm{Km}^{2}\end{array}$ & Superficie en \% \\
\hline \multirow{3}{*}{$\begin{array}{c}\text { Bassin } \\
\text { versant de } \\
\text { l'oued Beht }\end{array}$} & Faible & $0<$ Pente $<5$ & 4077,27 & 88,5 \\
\cline { 2 - 5 } & Moyenne & $5<$ Pente $<10$ & 59,89 & 1,3 \\
\cline { 2 - 5 } & Forte & $10<$ Pente $<20$ & 170,46 & 3,7 \\
\cline { 2 - 5 } & Très forte & Pente $>20$ & 299,46 & 6,5 \\
\cline { 2 - 5 } & Total & & 4607,08 & 100 \\
\hline
\end{tabular}

\section{Carte d'exposition des pentes}

La carte d'exposition des pentes permet de déterminer l'orientation des pentes avec leurs pourcentages ainsi que leur exposition dans le bassin 
versant. Elle est dominée principalement par les expositions Ouest et NordOuest (Fig. 8 et Tab. 4). L'importance de ces expositions est liée à la tectonique. En effet la dominance de l'exposition NW dans la partie amont du bassin, formée par le Plateau central et la partie occidentale du Causse moyen atlasique, est en relation avec l'affaissement tectonique par un réseau de failles qui se répartissent en trois familles principales: la famille de direction SW-NE, la famille de direction SE-NW et la famille de direction $\mathrm{N}$-S. L'effet de cette dernière est bien visible dans la partie Nord aval du bassin (Fig.8). Les rejeux de ces familles de failles au cours du Quaternaire expliqueraient non seulement la dominance de ces deux types d'exposition mais également la dissymétrie du réseau hydrographique qui est plus développé du côté droit de la vallée (Fig.8) que du côté gauche. Cette dissymétrie est liée à une migration globale de l'Oued Beht moyen vers l'Ouest sous l'effet des affaissements tectoniques qui se font vers l'Océan Atlantique. Ces affaissement expliquent aussi l'importance des dépôts plioquaternaires en allant vers l'Ouest, notamment dans la région de la Mamora.

Tableau 4. Exposition des pentes en pourcent.

\begin{tabular}{|c|c|c|c|c|c|c|c|c|}
\hline Exposition & Nord & Nord - Est & Est & Sud - Est & Sud & Sud - Ouest & Ouest & Nord - Ouest \\
\hline Pourcentage & 11,76 & 9,3 & 9,54 & 8,78 & 10,7 & 13,51 & 18,37 & 18,05 \\
\hline
\end{tabular}

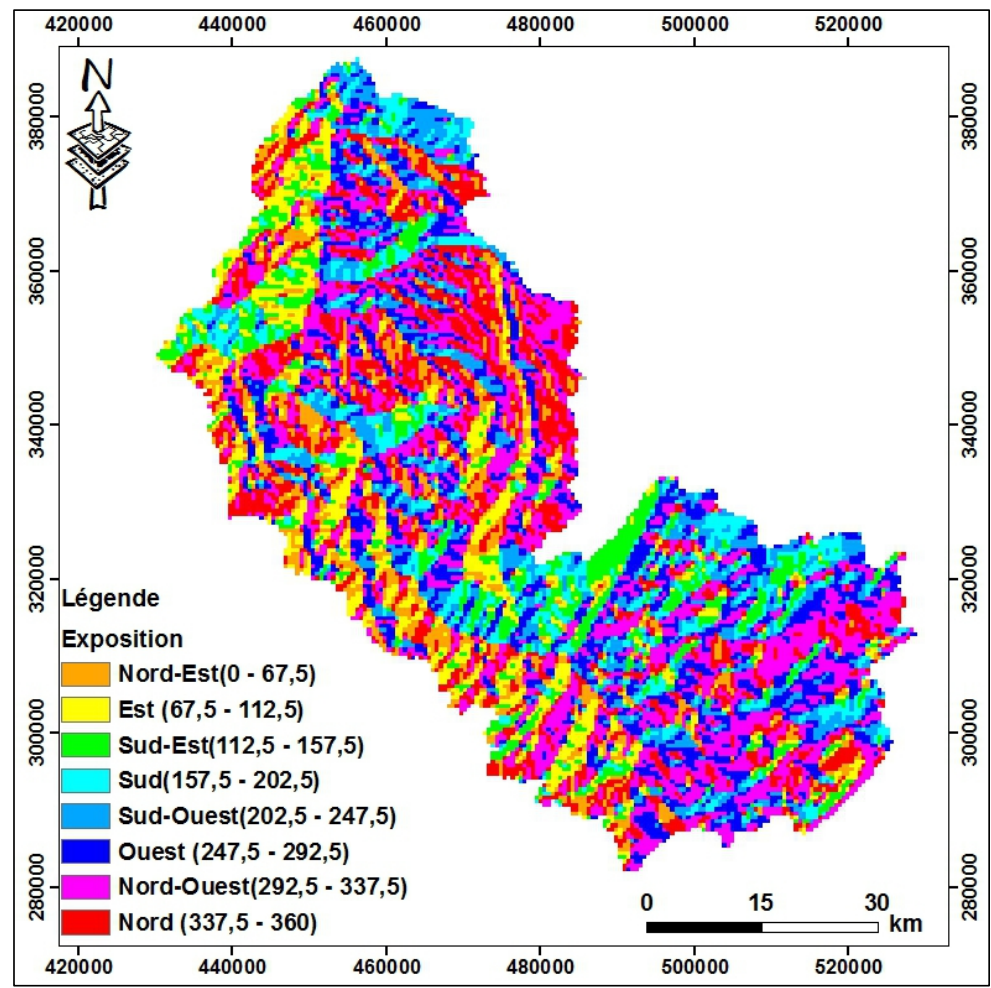

Figure 8. Cartes d'exposition des pentes. 


\section{Caractéristiques orographiques (indices de pentes)}

\section{Indice de pente de Roche}

L'indice de pente de Roche Ip caractérise la pente moyenne du bassin versant et se calcule à partir du rectangle équivalent. Il est égal à la somme des racines carrées des pentes moyennes de chaque élément partiel compris entre deux courbes de niveau, pondéré par la surface partielle qui lui est associée. Il s'exprime par:

$$
\text { Ip }=\frac{1}{\sqrt{\mathrm{Leq}}} \cdot \sum_{1}^{n} \sqrt{\text { ai. di }}
$$

$\mathrm{L}_{\mathrm{eq}}$ : Longueur du rectangle équivalent $=158,74 \mathrm{~km}=158740 \mathrm{~m}$,

ai : représente la fraction de la surface totale $\mathrm{A}$ du bassin versant comprise entre deux courbes de niveau voisines distantes de di=100m. Elle est donnée par la répartition hypsométrique.

di : désigne l'équidistance ou la dénivelée en $(\mathrm{m})$ entre deux courbes de niveau, di $=100 \mathrm{~m}$.

Pour le bassin versant de l'Oued Beht, l'Indice de pente de Roche Ip= 0,11 soit $11 \%$.

Tab. 5. Classes d'altitudes, surfaces partielles et dénivellation du bassin versant de l'Oued Beht.

\begin{tabular}{|c|c|c|c|c|c|}
\hline $\begin{array}{c}\text { Classe } \\
\text { d'altitude }\end{array}$ & $\begin{array}{c}\text { Surface entre } \\
\text { courbes de } \\
\text { niveau }\left(\mathrm{km}^{2}\right)\end{array}$ & Surface partielle ai & Dénivellation di (m) & ai *di & $\sqrt{\mathbf{a i} * \mathbf{d i}}$ \\
\hline $65-100$ & 1,56 & 0,00 & 35 & 0,01 & 0,11 \\
\hline $100-200$ & 154,61 & 0,03 & 100 & 3,36 & 1,83 \\
\hline $200-300$ & 299,88 & 0,07 & 100 & 6,51 & 2,55 \\
\hline $300-400$ & 375,91 & 0,08 & 100 & 8,17 & 2,86 \\
\hline $400-500$ & 314,21 & 0,07 & 100 & 6,83 & 2,61 \\
\hline $500-600$ & 251,34 & 0,05 & 100 & 5,46 & 2,34 \\
\hline $600-700$ & 319,51 & 0,07 & 100 & 6,94 & 2,63 \\
\hline $700-800$ & 286,90 & 0,06 & 100 & 6,23 & 2,50 \\
\hline $800-900$ & 374,26 & 0,08 & 100 & 8,13 & 2,85 \\
\hline $900-1000$ & 494,11 & 0,11 & 100 & 10,73 & 3,28 \\
\hline $1000-1100$ & 538,43 & 0,12 & 100 & 11,70 & 3,42 \\
\hline $1100-1200$ & 339,24 & 0,07 & 100 & 7,37 & 2,71 \\
\hline $1200-1300$ & 183,80 & 0,04 & 100 & 3,99 & 2,00 \\
\hline $1300-1400$ & 81,96 & 0,02 & 100 & 1,78 & 1,33 \\
\hline $1400-1500$ & 67,41 & 0,01 & 100 & 1,46 & 1,21 \\
\hline $1500-1600$ & 53,58 & 0,01 & 100 & 1,16 & 1,08 \\
\hline $1600-1700$ & 66,72 & 0,01 & 100 & 1,45 & 1,20 \\
\hline $1700-1800$ & 94,56 & 0,02 & 100 & 2,05 & 1,43 \\
\hline $1800-1900$ & 143,87 & 0,03 & 100 & 3,13 & 1,77 \\
\hline $1900-2000$ & 123,86 & 0,03 & 100 & 2,69 & 1,64 \\
\hline $2000-2100$ & 34,86 & 0,01 & 100 & 0,76 & 0,87 \\
\hline & & & & & \\
\hline
\end{tabular}




\begin{tabular}{|c|c|c|c|c|c|}
\hline $2100-2187$ & 3,19 & 0,00 & 87 & 0,06 & 0,25 \\
\hline Total & 4603,77 & 1 & & & 42,47 \\
\hline
\end{tabular}

\section{Indice de pente classique ou Indice de pente moyenne (\%)}

La pente moyenne est une caractéristique importante qui renseigne sur la topographie du bassin. Elle donne une bonne indication sur le temps de parcours du ruissellement direct, donc sur le temps de concentration tc, et influence directement le débit de pointe lors d'une averse (Musy, 2005). Elle gouverne la vitesse du ruissellement, qui à son tour détermine le pouvoir érosif et la capacité de transport de celui-ci (Young et al., 1996). Cette pente, qui dépend étroitement des conditions morpho-structurales dans le bassin hydrographique, détermine aussi le type dominant du style fluvial le long des lits fluviaux des cours d'eau actuels. Chaque style fluvial s'exprime, du point de vue morpho-sédimentaire, par des morphologies et des géométries bien définies des dépôts sédimentaires qui se mettent en place pendant les crues. A titre d'exemple, en cas ou cette pente est moyenne à faible, le style dominant est le style méandriforme avec des méandres libres. Ce style est caractérisé par des dépôts qui se mettent en place essentiellement dans des barres, dites de méandres, des plaines d'inondations bien développées et surtout par des méandres abandonnées (Leopold et Wolman, 1960 ; Bravard et Petit , 1997) qui se comportent comme des lacs plus au moins permanents selon les conditions climatiques.

L'Indice de pente classique $\mathrm{I}_{\mathrm{c}}$ (ou Indice de pente moyenne $\mathrm{Im} \mathrm{du}$ bassin versant) consiste à rapporter l'altitude entre les deux points extrêmes (Dénivelée totale du bassin versant en mètre $\mathrm{D}_{\mathrm{T}}=\mathrm{Hmax}-\mathrm{Hmin}$ ) à la longueur du bassin définie par la longueur du rectangle équivalent (Leq) :

$$
I c=\frac{\mathrm{DT}}{\mathrm{Leq}}=\frac{\mathrm{Hmax}-\mathrm{Hmin}}{\mathrm{Leq}}
$$

$\mathrm{Hmin}=65 \mathrm{~m}$ et $\mathrm{Hmax}=2187 \mathrm{~m}$ et $\mathrm{D}_{\mathrm{T}}=\mathrm{Hmax}-\mathrm{Hmin}=2122 \mathrm{~m}$

Par application de la formule, Ic du bassin versant de l'oued Beht est :

Ic $=(2187-65) / 158,74=2122 / 158,74=13,37$. Donc Ic $=\mathbf{1 3 , 3 7 \%}$. Il s'agit donc d'une pente modérée.

\section{Indice de pente global}

L'indice de pente global (Ig) est utilisé pour déterminer la dénivelée spécifique du bassin. Il permet de caractériser et de classer le relief du bassin versant. Dans une région de géomorphologie homogène, la pente diminue de l'amont vers l'aval, par conséquent, Ig décroît pour un même bassin lorsque la surface augmente. Toutefois, la longueur du rectangle équivalent étant généralement proche de celle du plus long cours d'eau, Ig reste voisin de la pente longitudinale (FAO, 1996). 
Sur la courbe hypsométrique, on prend les deux points tels que la surface supérieure et inférieure soit égale à $5 \%$ de l'aire totale $\mathrm{A}$ $\mathrm{du}$ bassin versant. On obtient les altitudes $\mathrm{H}_{5 \%}$ et $\mathrm{H}_{95} \%$ entre lesquelles s'inscrit $90 \%$ de A et la dénivelée globale $\mathrm{D}_{\mathrm{G}}=\mathrm{H}_{5 \%}-\mathrm{H}_{95 \%}$.

Pour le BV étudié, $\mathrm{H}_{5 \%}=1800 \mathrm{~m}, \mathrm{H}_{95 \%}=215 \mathrm{~m}$.

L'indice global de pente Ig est exprimé en $\mathrm{m} / \mathrm{km}$. Il est défini par la formule :

$$
I g=\frac{\mathrm{DG}}{\mathrm{Leq}}=\frac{\mathrm{H} 5 \%-\mathrm{H} 95 \%}{\mathrm{Leq}}
$$

- où, $D_{G}$ représente la dénivelée globale, exprimée en mètres, séparant les altitudes ayant approximativement $5 \%$ et $95 \%$ de la surface du bassin audessus d'elles. Ces altitudes sont déterminées sur la courbe hypsométrique. La dénivelée globale du bassin étudié est $\mathrm{D}_{\mathrm{G}}=1585 \mathrm{~m}$.

- Leq est la longueur du rectangle équivalent, exprimée en $\mathrm{km}$. Leq =158,74 $\mathrm{km}$.

Pour le bassin versant de l'Oued Beht, $\mathbf{I g}=\mathbf{9 , 9 8} \approx \mathbf{1 0} \mathbf{~ m} / \mathbf{k m}=\mathbf{0 , 0 1}$. Cette valeur reflète donc un relief modéré (Tab. 6).

Tableau 6. Classification du relief selon Ig par l'ORSTOM (Office de Recherche Scientifique de Territoire d'Outre Mer) pour des bassins versants de $25 \mathrm{~km}^{2}$ de surface.

\begin{tabular}{|c|c|}
\hline Type de relief & Indice de pente globale \\
\hline Relief très faible & $\mathrm{Ig}<0.002 \mathrm{~m} / \mathrm{km}$ \\
\hline Relief faible & $0.002<\mathrm{Ig}<0.005$ \\
\hline Relief assez faible & $0.005<\mathrm{Ig}<0.01$ \\
\hline Relief modéré & $0.01<\mathrm{Ig}<0.02$ \\
\hline Relief assez fort & $0.02<\mathrm{Ig}<0.05$ \\
\hline Relief fort & $0.05<\mathrm{Ig}<0.5$ \\
\hline Relief très fort & $0.5 \mathrm{~m} / \mathrm{km}<\mathrm{Ig}$ \\
\hline
\end{tabular}

Dans notre région d'étude, la relation entre l'indice de pente de Roche Ip et l'indice globale de pente Ig est : Ig=0,83.Ip ${ }^{2}$. Par ailleurs, cet indice Ig est simple et étroitement corrélé avec l'indice de pente de Roche (Ig $=0,8 \mathrm{Ip}^{2}$ ), avec un coefficient de corrélation de l'ordre de 0,99 (Laborde, 2009).

Apparemment la pente est modérée avec un relief accidenté qui domine sur la partie Sud et SE du terrain, ce qui à une influence directe sur la vitesse d'écoulement donc sur l'énergie cinétique produite, ce qui causera sans doute une érosivité très élevée sur tout les matériaux érodables dans la zone amont du bassin versant et plus précisément au niveau du Moyen Atlas et du Maroc central. 


\section{Dénivelée spécifique}

La dénivelée spécifique (Ds) permet de se prononcer sur le relief suivant la classification de l'Office de Recherche Scientifique d'Outre-Mer (ORSTOM). Elle représente le produit de l'indice de pente global (Ig) par la racine carrée de la superficie du bassin (A). Elle s'exprime en mètres et est indépendante, en théorie, de l'aire du bassin. Plusieurs classes de relief en fonction de Ds sont distinguées (Tab. 7). Elle est calculée par la formule :

$$
\text { Ds }=\text { Ig. } \sqrt{\mathbf{A}}
$$

Donc, Ds $=9,98 * \sqrt{4603,77}=677,15 ;$ Ds $=\mathbf{6 7 7 , 1 5} \mathbf{~ m}$.

Si Ig $=$ D/L et A $=$ L.1,

Alors, la Dénivelée spécifique devient :

$$
D s=\mathrm{D} \cdot \sqrt{\mathbf{1} / \mathbf{L}}
$$

La dénivelée spécifique ne dépend donc que de l'hypsométrie $\left(\mathrm{D}_{\mathrm{G}}=\mathrm{H}_{5 \%}\right.$ - $\left.\mathrm{H}_{95 \%}\right)$ et de la forme du bassin (1/L). Elle donne lieu à la deuxième classification de l'ORSTOM (Office de Recherche Scientifique de Territoire d'Outre Mer), indépendante des surfaces des bassins (Tab. 7).

Tableau 7. Classification du relief selon la méthode de l'ORSTOM.

\begin{tabular}{|c|c|c|}
\hline Classe & Type de relief & Ds \\
\hline R1 & Relief très faible & 10 \\
\hline R2 & Relief faible & $10-25$ \\
\hline R3 & Relief assez faible & $25-50$ \\
& & $50-100$ \\
\hline R4 & Relief modéré & $100-250$ \\
\hline R5 & Relief assez fort & $250-500$ \\
\hline R6 & Relief fort & $>\mathbf{5 0 0}$ \\
\hline R7 & Relief très fort & \\
\hline
\end{tabular}

La dénivelée spécifique du BV étudié de l'Oued Beht est Ds=677,15 $\mathrm{m}$. Sa valeur, $>500$, se trouve dans la classe $\mathbf{R} 7$ qui reflète un relief très fort d'où l'influence bien prononcée des massifs montagneux dans ce bassin versant (Tab. 7).

La valeur de Ds, traduit bien l'importance du volume montagneux et la forte incision du relief. Le bassin versant de l'oued Beht présente un relief très fort (R7) car : Ds $>500 \mathrm{~m}$.

L'utilisation du modèle numérique du terrain est très nécessaire à cause du gain de temps dans l'extraction automatique des indices relatifs au relief du bassin et à sa pente.

\section{Récapitulation des caractéristiques du bassin versant de l'Oued Beht}

Toutes les caractéristiques physiques du bassin versant de l'Oued Beht en amont du barrage El Kansera sont reprises dans le tableau récapitulatif (Tab. 10). 
Tableau 10. Caractéristiques physiographiques (physiques) du basin versant de l'Oued Beht en amont du barrage El Kansera.

\begin{tabular}{|c|c|c|c|c|}
\hline Paramètr & & Symboles & Unités & Valeurs \\
\hline \multicolumn{5}{|c|}{ Morphologie du bassin versant } \\
\hline \multicolumn{2}{|c|}{ Superficie } & $\mathrm{A}$ & $\mathrm{km}^{2}$ & 4603,77 \\
\hline \multicolumn{2}{|c|}{ Périmètre } & $\mathrm{P}$ & $\mathrm{km}$ & 421,43 \\
\hline \multicolumn{2}{|c|}{ Indice de Gravélius } & $\mathrm{K}_{\mathrm{G}}$ & -- & 1,74 \\
\hline \multicolumn{2}{|c|}{ Indice de forme d'Horton } & $\mathrm{K}_{\mathrm{H}}$ & -- & 0,16 \\
\hline \multicolumn{2}{|c|}{ Index de Forme } & $\mathrm{F}$ & -- & 0,42 \\
\hline \multicolumn{2}{|c|}{ Longueur du bassin versant } & $\mathrm{L}$ & $\mathrm{km}$ & 121,43 \\
\hline \multicolumn{2}{|c|}{ Largeur du bassin versant } & $\mathrm{B}$ & $\mathrm{km}$ & 51,43 \\
\hline \multirow{2}{*}{ Rectangle équivalent } & Longueur & Leq & $\mathrm{km}$ & 158,74 \\
\hline & Largeur & leq & $\mathrm{km}$ & 29 \\
\hline \multicolumn{5}{|c|}{ Relief } \\
\hline \multicolumn{2}{|c|}{ Altitude maximale } & Hmax & $\mathrm{m}$ & 2187 \\
\hline \multicolumn{2}{|c|}{ Altitude minimale } & Hmin & $\mathrm{m}$ & 65 \\
\hline \multicolumn{2}{|c|}{ Altitude moyenne } & $\mathrm{H}_{\text {moy }}$ & $\mathrm{m}$ & 878,82 \\
\hline \multicolumn{2}{|c|}{ Altitude médiane } & $\mathrm{H}_{50 \%}$ ou $\mathrm{H}_{\text {med }}$ & $\mathrm{m}$ & 900 \\
\hline \multicolumn{2}{|c|}{ Hauteur $5 \%$} & $\mathrm{H}_{5 \%}$ & $\mathrm{~m}$ & 1800 \\
\hline \multicolumn{2}{|c|}{ Hauteur $95 \%$} & $\mathrm{H}_{95 \%}$ & $\mathrm{~m}$ & 215 \\
\hline \multicolumn{2}{|c|}{ Pente moyenne } & $\mathrm{I}_{\mathrm{m}}$ & $\mathrm{m} / \mathrm{km}$ ou $\%$ & 13,38 \\
\hline \multicolumn{2}{|c|}{ Indice de pente de Roche } & Ip & -- & 0,11 \\
\hline \multicolumn{2}{|c|}{$\begin{array}{c}\text { Indice de pente classique ou Indice de pente } \\
\text { moyenne }\end{array}$} & $\mathrm{I}_{\mathrm{c}}$ ou Im & $\mathrm{m} / \mathrm{km}$ ou $\%$ & 13,37 \\
\hline \multicolumn{2}{|c|}{$\begin{array}{c}\text { Dénivelée totale du bassin versant } \mathrm{D}_{\mathrm{T}}= \\
\text { Hmax }-\mathrm{Hmin}\end{array}$} & $\mathrm{D}_{\mathrm{T}}$ & $\mathrm{m}$ & 2122 \\
\hline \multicolumn{2}{|c|}{ Dénivelée $\mathrm{D}=\mathrm{H}_{5 \%}-\mathrm{H}_{95 \%}$} & $\mathrm{D}$ & $\mathrm{m}$ & 1585 \\
\hline \multicolumn{2}{|c|}{ Indice de pente global } & $\mathrm{Ig}$ & -- & 0,01 \\
\hline \multicolumn{2}{|c|}{ Dénivelée spécifique } & Ds & $\mathrm{m}$ & 677,15 \\
\hline
\end{tabular}

\section{Conclusion}

Le bassin versant de l'Oued Beht en amont du barrage El Kansera a été étudié à partir de son modèle numérique de terrain (MNT) pour l'extraction automatique, à l'aide des logiciels SIG, de ses caractéristiques géomorphologiques qui influencent l'écoulement de ses eaux de surface. L'évaluation de ces paramètres a permis la réalisation de nombreuses cartes thématiques et leur interprétation.

Le bassin versant étudié s'étale sur une superficie d'environ 4603,77 $\mathrm{km}^{2}$ et présente un périmètre de $421,43 \mathrm{~km}$. Il est caractérisé par un relief modéré à fort et une forme allongée $(\mathrm{KG}=1,74)$ suivant une direction SudEst-Nord-Ouest parallèlement au sens de l'écoulement général des eaux. Cette forme, liée aux conditions morpho-structurales, favorise une érosion linéaire et régressive et les faibles débits de pointes de crues en raison du temps élevé de concentration ou d'acheminement des eaux jusqu'à l'exutoire. Ce type de bassin versant devrait présenter des plaines 
d'inondation peu développées, des méandres avec de faible à moyen rayon de courbure, peu de méandres abandonnés et un style fluvial dominé par le style faiblement sinueux à méandriforme avec localement une tendance au tressage. Ce type de bassin devrait également connaître très peu de cas d'inondation des localités riveraines même pendant les périodes des hautes eaux. La circularité $\mathrm{Rc}=0,33$, l'indice de forme d'Horton, $\mathrm{KH}=0,16$, l'Index de Forme $\mathrm{F}=0,42$ confirment la forme allongée du bassin versant et impliquent une production d'écoulements torrentiels pendant les pluies orageuses qui s'abattent dans le bassin versant. La forme allongée du bassin est liée à une instabilité tectonique qui est confirmée par la dissymétrie de la vallée beaucoup plus large du côté droit que du côté gauche à cause de la migration de l'oued vers l'Ouest sous l'effet de l'affaissement de cette zone.

De point de vue hypsométrique, les zones de hautes altitudes se répartissent dans le SE du bassin et les zones de basses altitudes se trouvent au NW. Cette répartition de l'altitude est liée à la topographie du bassin versant composée d'unités plus au moins étagées, correspondant, géomorphologiquement, à des plateaux disposés en marches d'escaliers et passant vers l'aval à des plaines. Ces dispositifs topographique et géomorphologique sont l'expression d'un compartimentage tectonique en blocs de plus en plus affaissés depuis le SE vers le NW déterminant ainsi une structure géologique en demi graben.

La courbe hypsométrique rappelle celle d'un bassin versant qui a déjà dépassé l'état de maturité et d'équilibre et a atteint par conséquent un stade relativement développé d'érosion. Nous pouvons d'ores et déjà avancer qu'au niveau de ce bassin versant, le pouvoir de l'érosion fluviale était très développé. L'érosion s'est faite principalement de manière mécanique comme le prouve d'ailleurs la composition des dépôts modernes du fond de la vallée composés de sédiments détritiques terrigènes. Cette érosion exprime une dégradation des conditions bioclimatiques qui sont devenues de plus en plus sévères en s'exprimant alors par une raréfaction du couvert forestier liée à la conjugaison d'une tendance à l'aridification du climat actuel et une intensification de l'action anthropique dans le bassin versant.

Les contrastes orographiques entre l'amont et l'aval d'une part et au sein d'une même zone, pouvant correspondre à un sous bassin versant, d'autre part sont étroitement liés aux conditions géologiques et géomorphologiques : la lithologie des terrains et leur structure géologique et les types de relief structuraux qui vont depuis des reliefs de type appalachien dans les formations géologiques très tectonisées du socle paléozoïque, à des reliefs de structure de type monoclinal à sub-tabulaire dans les formations géologiques mésozoïques et cénozoïques de couverture. Ce facteur, favorisant l'érosion hydrique, est à tenir en compte pour atténuer les 
problèmes d'envasement du barrage El Kansera situé en aval du bassin versant.

Les dimensions du rectangle équivalent (longueur, $\mathrm{L}=158,74 \mathrm{~km}$, largeur, $1=29 \mathrm{~km}$ ) traduisent que la longueur est 5,47 fois plus grande que la largeur. Les altitudes varient de $65 \mathrm{~m}$ à plus de $2187 \mathrm{~m}$ avec une moyenne de $878,82 \mathrm{~m}$ et une altitude médiane de $900 \mathrm{~m}$. La pente moyenne du bassin versant de l'Oued Beht est modérée avec 13,38\%, ce qui présente une grande durée de concentration des eaux de ruissellement dans les affluents, favorisant ainsi leur infiltration surtout lorsque les formations lithologiques présentent une grande perméabilité. Les pentes les plus accusées, très fortes à fortes, se concentrent dans la partie SW dans le Maroc central et dans le SE du bassin versant dans le Causse du Moyen Atlas. Les pentes faibles à très faibles correspondent à la partie aval du bassin versant juste avant le barrage El Kansera dans le Sillon sud rifain septentrional. Ces pentes sont dominées principalement par des orientations Ouest et Nord-Ouest en relation avec le réseau de fracturation.

Enfin, il faut souligner que, malgré tous ses mérites et les remarquables contributions des modèles numériques de terrain, bien qu'ils constituent une donnée très puissante d'analyse, l'informatique ne peut suffire à elle seule à résoudre tous les problèmes géologiques, géomorphologiques, topographiques et d'aménagement. Il faudrait des démarches complémentaires s'appuyant sur la réalité du terrain.

\section{References:}

1. Abbassi H., 2004. Utilisation de l'outil SIG pour l'aménagement des bassins versants de Oued «Greb» et Oued «Rouriche » à l'Ariana. Mémoire de PFE. ESIER de Medjez El Beb. 67p.

2. ABHS (Agence du Bassin Hydraulique de Sébou), 2011. Etude d'actualisation du plan directeur d'aménagement intégré des ressources en eau du bassin hydraulique de Sebou. Note de synthèse, $103 p$.

3. Bannister E. N., 1980. Hypsometries of Michigan's Southeastern Lake Plain. Journal of Great Lakes Research, Vol. 6, Issue 2, 154163.

4. Beaudet G., 1969. Le Plateau central marocain et ses bordures. Etude géomorphologique. Inframar, Rabat, 480p.

5. Benyassine M. et Dekayir A., 2013. Application du SIG et de l'imagerie radar à l'analyse morphologique et minéralogique du bassin versant de la Haute Moulouya (Maroc). Geo-Eco-Trop., 37, 2, 227-242.

6. Biswas S., Sudhakar S. et Desai V. R., 1999. Prioritization of sub watersheds based on morphometric analysis of drainage basin. A 
remote sensing and GIS approach. Journal of Indian Society of Remote Sensing, 27(3), 155-166.

7. Bravard J.P. \& Petit F., 1997. Les cours d'eau, dynamique du système fluvial. Armand Colin ed., Paris, 221p.

8. Cartier L. \& Leclerc S., 1964. Rivière Eaton: caractéristiques topographiques $d u$ bassin versant. Ministère des Richesses Naturelles du Québec, Service d'hydrométrie, Québec.

9. Collin N., 2009. Geodynamic framework of large volcanic fields highlighted by SRTM DEMs: Method evaluation and perspectives exampled on three areas from the Cameroon Volcanic Line. J. Volcano and Geoth Res., 187, 13-25.

10. FAO, 1996. Crues et apports. Manuel pour l'estimation des crues décennales et des apports annuels pour les petits bassins versants non jaugés de l'Afrique sahélienne et tropicale sèche. Bulletin FAO d'irrigation et de drainage, 54, 244p. http://www.fao.org/docrep/w2570f/w2570f00.htm\#Contents.

11. Gregory K.J., Walling D.E., 1973. Drainage basin form and process: a geomorphological approach. John Wiley, New York, 456p.

12. Horton R. E., 1945. Erosional development of streams and their drainage basins (hydrophysical approach to quantitative morphology). Geological Society of America Bulletin, 56, 275-390.

13. Laborde J.P., 2009. Eléments d'hydrologie de surface. Cours dispensés au Cycle ingénieur et Master Hydroprotech. Ecole Polytechnique, Université de Nice - Sophia Antipolis, 192p.

14. Leopold L.B. \& Wolman M.G., 1960. River meanders. Bulletin of the Geological Society of America, 71, 769-94.

15. Llamas J., 1993. Hydrologie générale : Principes et Applications. Edition Gaetan morin, Quebec, 527p.

16. Li Z., Zhu Q., Gold C., 2004. Digital Terrain Modeling: Principles And Methodology. CRC Ed., 323p.

17. Maune D.F., Koff S.M., Crawford C.A., Zervas C.E., 2001. Introduction. In Maune DF (ed). Digital Elevation Model Technologies and Applications: the DEM user's Manual. American Society for Photogrammetry and Remote Sensing: Bethesda, MD, 134.

18. Michel P., 1973. Les bassins des fleuves Sénégal et Gambie : étude géomorphologique. Doctorat d'Etat, Mémoires ORSTOM, $\mathrm{n}^{\circ}$ 63, 752 p, 3 tomes, Paris, Edition de 1'Office de la Recherche Sciences et Techniques Outre-Mer.

19. Miller V.C., 1959. A quantitative geomorphic study of drainage basin characteristics in the clinch mountain area, Virginia and Tennessee. 
Project NR 389-042, Technical Report 3, New York, Department of geology, Columbia University.

20. Morell M., Thébé B. et L’Hote Y., 1999. Acquisition et constitution d'une information hydrologique de base. Editions H.G.A., Bucarest, 203p. ISBN 973-98954-1-7.

21. Moţoc M., Munteanu S., Băloiu V., Stănescu P., Mihai G., 1975. Eroziunea solului şi metodele de combatere. Editura Ceres, Bucureşti, 301p.

22. Musy A. \& Laglaine V., 1992. Hydrologie générale. Cours polycopié, section génie rural, environnement, mensuration, EPFL.

23. Musy A., 2005. Cours d'hydrologie générale. Laboratoire d'hydrologie et Aménagements (HYDRAM), Ecole Polytechnique Fédérale, Lausanne (Suisse).

24. ORE, 1998. Bilan hydrologique du bassin versant du Bras Panon. Opération 3111100000. Direction de l'Agriculture et de la Forêt et Conseil General de la Réunion, Rapport de synthèse, 170p.

25. Reddy G.P.O., Maji A.K., Gajbhiye K.S., 2004. Drainage morphometry and its influence on landform characteristics in a basaltic terrain, Central India - a remote sensing and GIS approach. Applied Earth Observation and Geoinformation, 6, 1-16.

26. Roche M., 1963. Hydrologie de surface. Edition Gauthier-Villars et ORSTOM, Paris.

27. Singh V.P., 1992. Elemental hydrology. Prentice Hall, Englewood Cliffs, New Jersey, 973p.

28. Sreedevi P., Subrahmanyam K., Ahmed S., 2005. The significance of morphometric analysis for obtaining groundwater potential zones in a structurally controlled terrain. Environmental Geology, 47, 412-420.

29. Strahler A.N., 1952. Hypsometric (area-altitude curve) analysis of erosional topography. Geological Society of America Bulletin, 63, 1117-1141.

30. Strahler A. N., 1964. Quantitative geomorphology. In: Handbook of Applied Hydrology (ed. by V. T. Chow), Section 4-II. McGraw-Hill, New York, USA.

31. Veltri M., Veltri P. et Maiolo M., 1996. On the fractal dimension of natural channel network. Journal of Hydrology, 187, 137-144.

32. Young W.J., Marston F.M. et Davis J.R., 1996. Nutrient exports and land use in Australian catchments. Journal of environmental management, $47,183 p$.

33. Zhou L., 2009. Longitudinal and transverse profiles of hilly and mountainous watersheds in Japan. Geomorph., 111, 17-26. 